\title{
Regulation of miR-155 affects pancreatic cancer cell invasiveness and migration by modulating the STAT3 signaling pathway through SOCS1
}

\author{
CHEN HUANG ${ }^{1-3^{*}}$, HAIDONG LI ${ }^{1,4^{*}}$, WEIDONG WU ${ }^{1 *}$, TAO JIANG ${ }^{1}$ and ZHENGJUN QIU ${ }^{1-3}$ \\ ${ }^{1}$ Department of General Surgery, Affiliated First People's Hospital, School of Medicine, Shanghai Jiao Tong University; \\ ${ }^{2}$ Shanghai Key Laboratory of Pancreatic Disease; ${ }^{3}$ Pancreatic Cancer Center of Shanghai Jiaotong University, \\ Shanghai 200080; ${ }^{4}$ Department of General Surgery, Shanghai Tongji University \\ Affiliated East Hospital, Shanghai 200120, P.R. China
}

Received May 1, 2013; Accepted May 31, 2013

DOI: 10.3892/or.2013.2576

\begin{abstract}
In the present study, we investigated the effects of miR-155 on pancreatic cancer cell invasion and migration in vitro, underlying gene expression, expression of miR-155 and its target genes in pancreatic cancer tissues, and their association with metastasis and clinical stage. miR-155 mimics and an inhibitor were transfected into Panc-1 and Capan-2 cells in order to regulate the expression of miR-155. qPCR and western immunoblotting were performed in order to detect gene expression. Transwell assays were performed to characterize the invasion and migration of pancreatic cancer cells in vitro. Immunohistochemical analysis and in situ hybridization were used to detect the expression of protein and microRNA in pancreatic cancer tissue. miR-155 mimics and an inhibitor upregulated and downregulated, respectively, the expression of miR-155 in pancreatic cancer cells. The invasion and migration of pancreatic cancer cells increased or decreased along with miR-155 expression in vitro. Suppressor of cytokine signaling 1 (SOCS1) protein expression was upregulated when miR-155 was inhibited and downregulated when miR-155 was increased. However, the expression of P-signal transducer and activator of transcription-3 (STAT3) was synchronized with that of miR-155. Transcription of SOCS1 and STAT3 was unchanged by miR-155 regulation. miR-155 expression was high in pancreatic cancer tissues and SOCS1 expression was high in tumor-adjacent tissues. There was no relationship between these genes in cancer and tumor-adjacent tissues. In addition,
\end{abstract}

Correspondence to: Dr Chen Huang or Professor Zhengjun Qiu, Department of General Surgery, Affiliated First People's Hospital, School of Medicine, Shanghai Jiao Tong University, No. 100 Haining Road, Shanghai 200080, P.R. China

E-mail: richard-hc@hotmail.com

E-mail: qiuwryb@online.sh.cn

${ }^{*}$ Contributed equally

Key words: pancreatic cancer, invasion, miR-155, STAT3
miR-155 expression was associated with lymph node metastasis and clinical stage. In conclusion, miR-155 plays an important role in the regulation of pancreatic cancer cell invasion and migration by modulating the STAT3 signaling pathway and reducing SOCS1 expression in pancreatic cancer cells.

\section{Introduction}

microRNAs (miRNAs) are molecules, 22 nucleotides long, that inhibit gene expression in animals and plants. Mounting evidence indicates that miRNAs are key regulators of human diseases such as cancer (1).

Pancreatic cancer is a deadly malignancy with a 5-year survival rate of $\sim 5 \%$; it is the fourth most common cause of cancer-related mortality in the Western world (2). The molecular mechanisms responsible for pancreatic cancer development remain unknown and there are no established guidelines for prevention. Recent studies have revealed a relationship between altered miRNA expression and pancreatic cancer $(3,4)$.

The miR-155 locus is located within a region known as the B-cell integration cluster (BIC) (3), which was originally thought to be a proto-oncogene associated with lymphoma (5). miR-155 is overexpressed in various solid tumors, including breast, lung, colon and thyroid cancers, where it functions as an oncogenic miRNA (6-9). Reports have also shown that many miRNAs including miR-155 are differentially expressed in pancreatic cancer $(10,11)$. High expression of miR-155 is correlated with poor prognoses of pancreatic cancer (12). miR-155 promotes pancreatic cancer development and mammary gland epithelial cell migration and invasion by targeting TP53INP1 and RhoA, respectively $(13,14)$. These lines of evidence are consistent with the notion that miR-155 plays an important role in the development of pancreatic cancer.

Suppressor of cytokine signaling 1 (SOCS1) is a tumor suppressor that normally functions as a negative feedback regulator of Janus activated kinase (JAK)/signal transducer and activator of transcription-3 (STAT3) signaling (15). It is a target gene of miR-155 in breast cancer (16). We found that STAT3 signaling was overactivated in pancreatic cancer and 
that it promoted invasion and metastasis $(17,18)$. However, the relationship between miR-155 overexpression and overactivation of STAT3 signaling in pancreatic cancer is unknown.

In the present study, we utilized miR-155 mimics and an inhibitor to regulate miR-155 expression. Migration and invasion in vitro were assessed, and SOCS1 expression and activation of STAT3 were detected. In situ hybridization and immunohistochemical analysis in tissue microarrays were performed to analyze the correlation of miR-155 and SOCS1 expression with various clinicopathologic factors.

\section{Materials and methods}

Cell culture and transient transfection. Human pancreatic cancer cell lines Panc-1 and Capan-2 were obtained from the American Type Culture Collection (Manassas, VA, USA) and cultured with Dulbecco's modified Eagle's medium (DMEM) supplemented with $10 \%$ fetal bovine serum (FBS) and penicillin/streptomycin at $37^{\circ} \mathrm{C}$ in a $5 \% \mathrm{CO}_{2}$ incubator. Panc- 1 and Capan-2 cells $\left(1 \times 10^{6}\right)$ were seeded into each well of 6 -well plates and transfected with miR-155 mimics and anti-miR-155. Cognate control RNAs were used as negative controls. Transfection was performed using Lipofectamine ${ }^{\circledR} 2000$ (Invitrogen) according to the manufacturer's instructions, and miR-155 mimics or antisense oligonucleotides were mixed with Lipofectamine 2000. After $48 \mathrm{~h}$, the cells were assayed. The sequences of miR-155 mimics were 5'-UUAAUGCUAAUC GUGAUAGGGGU-3' and 5'-CCCUAUCACGAUUAGCAU UAAUU-3'; the inhibitor sequence was 5'-ACCCCUAUCACG AUUAGCAUUAA-3'.

Invasion and migration assays. The cell invasion assay was performed in a specialized invasion chamber that included a 24-well tissue culture plate and 12-cell culture inserts (both from Corning). The inserts contained an $8-\mu \mathrm{m}$ pore polycarbonate membrane. A thin layer of basement membrane matrix (1:3 dilution; BD Biosciences) coated each well. Briefly, medium supplemented with $10 \%$ FBS was added to the lower chamber as a chemo-attractant. After reaching 60-70\% subconfluence, pancreatic cancer cells were trypsinized, re-suspended in DMEM, and $\sim 5 \times 10^{4}$ cells were added to each upper compartment.

After $48 \mathrm{~h}$ of incubation at $37^{\circ} \mathrm{C}$, the non-invasive cells and membranes were removed from the upper surface using a moist cotton swab. Invasive cells on the lower surface of the membrane were stained for $20 \mathrm{~min}$ and rinsed several times with distilled water. Invasiveness was quantified by selecting 5 different views (x400) and calculating the number of invading cells.

The cell migration assay was performed as the invasion assay, but the basement membrane matrix was not used and the cell seeding number was $8 \times 10^{4}$.

Quantitative real-time reverse transcription-polymerase chain reaction ( $q R T-P C R$ ). Total RNA was isolated from Panc-1 and Capan-2 cells using TRIzol reagent (Invitrogen). The RNA was then purified using an RNeasy Mini kit (Qiagen) according to the manufacturer's instructions. The miR-155, miR-21 and miR-210 levels were quantified by quantitative reverse transcription-PCR (qRT-PCR) using SYBR-Green assay kits (Genecopoeia), with U6 small nuclear RNA as an internal normalized reference. SOCS1 and STAT3 mRNA levels were determined using the forward and reverse primers with $\beta$-actin as an internal reference. Specific primers for the PCR reaction were as follows: SOCS1, 5'-GAGGGAGC GGATGGGTGTA-3' (forward) and 5'-GAGGTAGGAGGT GCGAGTTCAG-3' (reverse); STAT3, 5'-CCAAGGAGGAGG CATTCG-3' (forward) and 5'-ACATCGGCAGGTCAATGG-3' (reverse); $\beta$-actin, 5'-AGTTGCGTTACACCCTTTC-3' (forward) and 5'-CACCTTCACCGTTCCAGT-3' (reverse). Relative miRNA or mRNA expression of target genes, following normalization to an endogenous sequence, was calculated by the $\Delta \Delta \mathrm{Ct}$ method. miRNAs or mRNAs upregulated or downregulated 1-fold were identified as being significantly altered.

Protein extraction and western immunoblotting. Cells were harvested $48 \mathrm{~h}$ after transfection and lysed in radioimmunoprecipitation assay buffer (Beyotime, Haimen, Jiangsu, China) containing $1 \mathrm{mmol} / \mathrm{l}$ phenylmethanosulfonyl fluoride on ice for $15 \mathrm{~min}$. Protein concentration was determined with a BCA protein assay kit (Beyotime). Lysates were mixed with SDS-PAGE sample loading buffer and boiled for $5 \mathrm{~min}$. Total cellular protein $(50 \mu \mathrm{g})$ was resolved on 8 or $10 \%$ SDS-polyacrylamide gels and transferred to nitrocellulose membranes. The membranes were stained with $0.5 \%$ Ponceau S containing 1\% acetic acid to verify equal loading and transfer efficiency. The membranes were blocked in $5 \%$ bovine skim milk overnight and with primary antibody overnight at $4^{\circ} \mathrm{C}$. After washing in TBS, the membranes were incubated with peroxidase-conjugated secondary antibody for $1.5 \mathrm{~h}$ at room temperature. Enhanced chemiluminescence reagent from Millipore (Billerica, MA, USA) was used to detect positive protein bands. The primary antibodies were as follows: SOCS1 (1:1,000; Abcam, Cambridge, MA, USA), STAT3 $(1: 1,000)$ and P-STAT3 $(1: 2,000$; both from Cell Signaling Technology Danvers, MA, USA); and $\beta$-actin (1:1,000; Biomart, Shanghai, China). Secondary antibodies included peroxidase-conjugated Affinipure goat anti-mouse or anti-rabbit IgG (Jackson ImmunoResearch; West Grove, PA, USA).

Immunohistochemistry. Pancreatic cancer and tumor-adjacent tissue chips were purchased from Shanghai Outdo Biotech Co. (Shanghai, China); each point was $1.5 \mathrm{~mm}$ in diameter and $4 \mu \mathrm{m}$ thick. The chip was deparaffinized in xylene and rehydrated in successive washes of ethanol, and then heated in a microwave oven at medium power for $8 \mathrm{~min}$ in citrate buffer ( $\mathrm{pH} \mathrm{6.0)} \mathrm{for} \mathrm{heat-induced} \mathrm{epitope} \mathrm{retrieval.} \mathrm{Endogenous}$ peroxidase activity was blocked, followed by non-specific binding of the primary antibody, target protein localization with the first antibody, visualization with the secondary antibody, and the color reaction. The primary antibodies included SOCS1 $(1: 1,000)$.

Stained tumor cells and paraffin sections were reviewed and scored using light microscopy performed by a pathologist blinded to the treatment group. Positivity of the stained tumor cells on coverslips and paraffin sections was defined by staining intensity and the percentage of positive cells. The staining intensity of SOCS1 expression was classified 

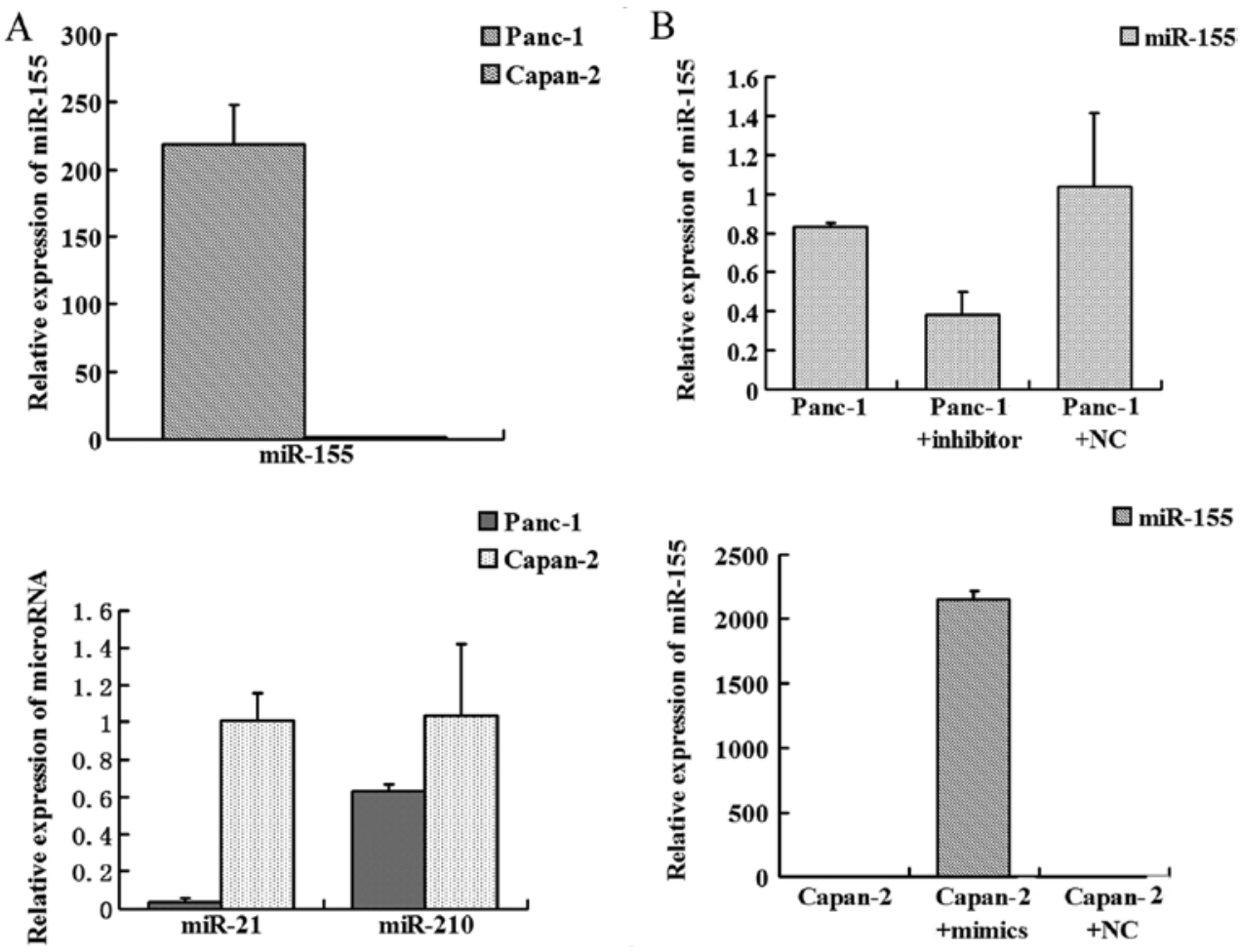

Figure 1. Expression of miR-155, miR-210 and miR-21 in Panc-1 and Capan-2 cells. We assessed the expression of three microRNAs which were reported to be associated with tumor invasion and high expression in pancreatic cancers. (A) miR-155 expression was much higher in the Panc-1 cells than that in the Capan-2 cells while miR-21 expression was lower in the Panc-1 cells than that in the Capan-2 cells, and there was no difference in the expression of miR-210 in both cell types. (B) miR-155 mimics upregulated miR-155 expression in Capan-2 cells; the inhibitor knocked down miR-155 expression in Panc-1 cells.

semi-quantitatively into negative and weak, moderate, and strong positivity $(0,+,++$ and +++ , respectively).

In situ hybridization of miRNAs. In situ hybridization of miR-155 was performed on tissue chip sections. The sequence of miR-155 probe was ACCCCTATCTCGATTAGCATT AA-HRP. Sections were deparaffinized in xylene, rehydrated in successive washes of DEPC-treated water through a graded series of ethanol (100, 70, 50 and 25\%), and left in PBS for 10 min. After permeabilization with $0.1 \%$ Triton X-100 in PBS for $10 \mathrm{~min}$, the sections were washed in PBS ( 2 x $5 \mathrm{~min})$ and treated with Proteinase $\mathrm{K}(1 \mu \mathrm{g} / \mathrm{ml}$ in $50 \mathrm{mmol} / 1$ EDTA, $\mathrm{pH} 8.0,0.1 \mathrm{~mol} / 1$ Tris- $\mathrm{HCl}$ ) for $5 \mathrm{~min}$ at $37^{\circ} \mathrm{C}$, followed by washing in PBS ( 3 x 5 min). Mercury Locked Nucleic Acid (LNA) miRNA detection probes (Fudan Biotechnology Co., Shanghai, China) were used; hsa-miR-155 (40 nM in a formamide-free ISH buffer). Probes were denatured by heating to $95^{\circ} \mathrm{C}$ for $5 \mathrm{~min}$ and $50 \mathrm{ml}$ of probe mixture was hybridized with the tissue sections in a hybridizer at $37^{\circ} \mathrm{C}$ for $60 \mathrm{~min}$. The slides were then placed at RT in $5 \mathrm{X}$ saline-sodium citrate (SSC) (Invitrogen) and washed for $5 \mathrm{~min}$ at $55^{\circ} \mathrm{C}$ in $5 \mathrm{X} \mathrm{SSC}$ (1 wash), 1X SSC ( 2 washes) and 0.5X SSC ( 2 washes). After washing in TBS, sections were blocked with blocking buffer and incubated for $30 \mathrm{~min}$. Slides were then incubated for 120 min in TBS with HRP-conjugated anti-DIG (diluted 1:500 in blocking solution; Roche). After washing in TBS ( 2 x 5 min), the DAB color reaction was performed.

Positivity of stained tumor cells on coverslips and paraffin sections was defined by staining intensity and the percentage of positive cells as in the immunohistochemistry experiment.
Statistical analysis. Statistical analyses were performed using SPSS 13.0 software (SPSS, Chicago, IL, USA). The data are expressed as means \pm SD when possible and were analyzed with the Student-Newman-Keuls test to determine statistical significance. $\mathrm{P}<0.05$ was considered statistically significant. Correlations were calculated using Spearman's $r$ test (two-sided) unless otherwise specified. P-values were not adjusted for multiple testing. Categorical variables were assessed by the Chi-square test.

\section{Results}

Regulation of miR-155 expression in Panc-1 and Capan-2 cells. miR-155, miR-210 and miR-21 have been reported to be associated with tumor invasion and are highly expressed in pancreatic cancers (5-9). We determined expression of these microRNAs in Panc-1 and Capan-2 cells. No difference in miR-210 expression was noted while miR-21 expression was higher in the Capan-2 cells when compared to that in the Panc-1 cells. miR-155 expression was much higher in the Panc-1 cells than that in the Capan-2 cells (Fig. 1A). qRT-PCR revealed that miR-155 mimics upregulated miR-155 expression in Capan-2 cells and the miR-155 inhibitor successfully knocked down miR-155 expression in Panc-1 cells (Fig. 1B).

Invasion and migration ability and miR-155 modulation in pancreatic cancer cells. We assessed changes in invasion and migration ability of Panc- 1 and Capan-2 cells after regulation of miR-155 expression by using Transwell assays. Upregulation of miR-155 expression in Capan-2 cells enhanced 


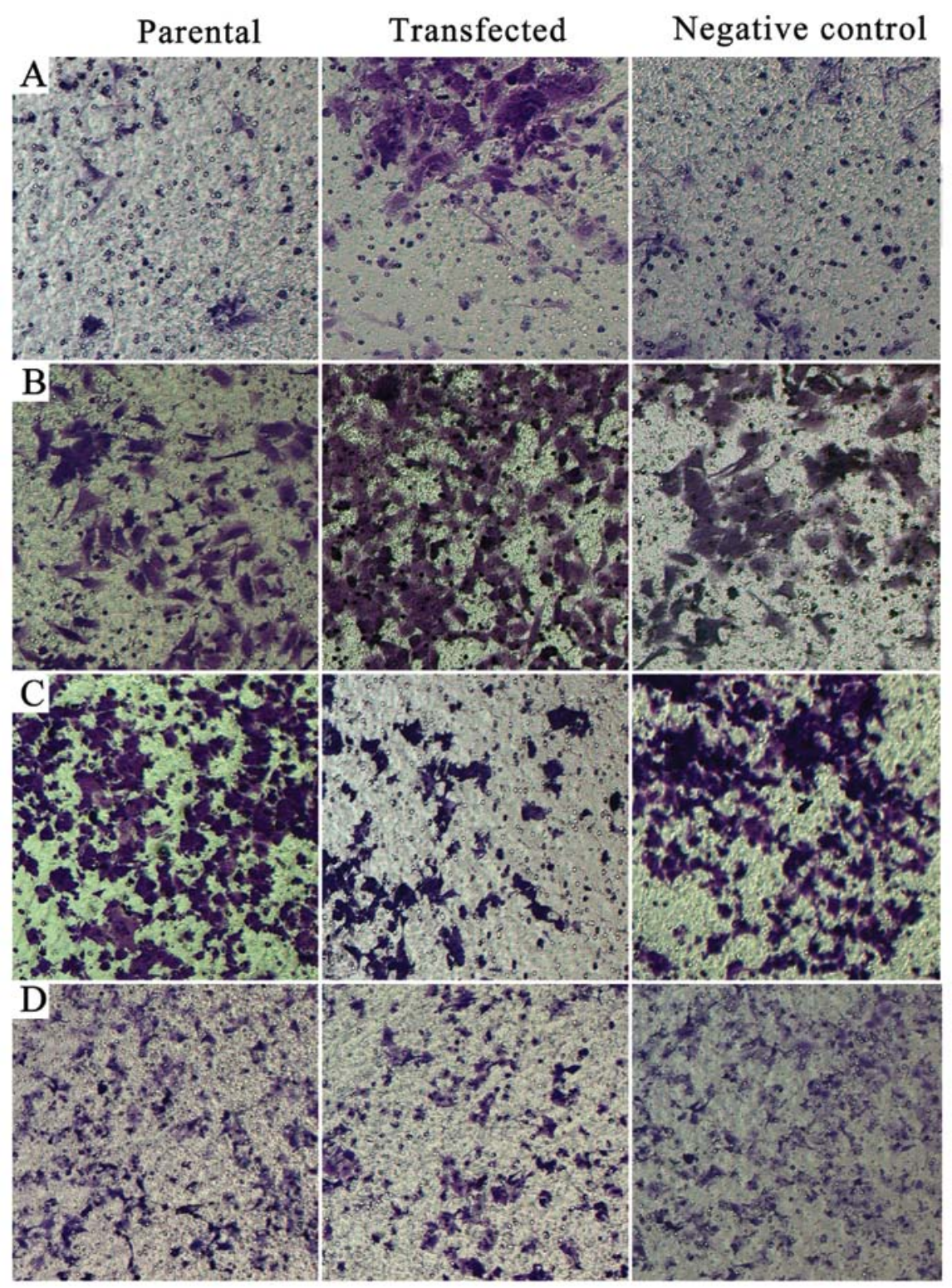

Figure 2. Invasion and migration of pancreatic cancer cells in vitro. (A) Changes in invasive ability following upregulation of expression of miR-155 in Capan-2 cells. (B) Changes in migratory ability following upregulation of expression of miR-155 in Capan-2 cells. (C) Changes in invasive ability following knockdown of expression of miR-155 in Panc-1 cells. (D) Changes in migratory ability following knockdown of expression of miR-155 in Panc-1 cells.

invasion and migration ability $(\mathrm{P}=0.0002, \mathrm{P}=0.0001)$ (Figs. 2A and $\mathrm{B}$; 3A and $\mathrm{B}$ ), and knockdown of miR-155 expression in Panc-1 cells inhibited invasion and migration ability ( $\mathrm{P}=0.0005, \mathrm{P}=0.0002$ ) (Figs. $2 \mathrm{C}$ and $\mathrm{D} ; 3 \mathrm{C}$ and $\mathrm{D}$ ).

Expression of SOCS1 and STAT3 and activation of STAT3 following regulation of miR-155. We determined SOCS1 gene expression in miR-155-regulated cells by qRT-PCR and western blotting. The data revealed that SOCS1 and STAT3 mRNA expression did not differ in the transfected cells when compared with the parental and control cells (Fig. 4A). However, at the protein level, SOCS1 expression was increased by miR-155 knockdown and decreased by miR-155 upregulation in Panc-1 cells. P-STAT3 protein was decreased by miR-155 knockdown in Panc-1 cells and was increased by miR-155 upregulation in Capan-2 cells (Fig. 4B).
Expression of miR-155 and SOCS1 in pancreatic cancer and tumor-adjacent tissues. We detected expression of miR-155 and SOCS1 in pancreatic cancer and tumor-adjacent tissues in tissue chips by in situ hybridization and immunohistochemistry. The rate of miR-155-positive expression in the pancreatic cancer tissues was $81.25 \%(65 / 80)$, and the rate of strong-positive expression was 10\% (10/80). However, in tumor-adjacent tissues, the rate of miR-155-positive expression was $71.25 \%$ (57/80) and the rate of strong-positive expression was $1.25 \%(1 / 80)$. Statistical analyses showed that miR-155-positive expression in pancreatic cancer tissues was significantly higher than that in tumor-adjacent tissues ( $\mathrm{P}=0.0001)$ (Fig. 5A) (Table I). The positive expression rate of SOCS1 in pancreatic cancer tissues was $37.5 \%(30 / 80)$ and $65 \%(52 / 80)$ in tumor-adjacent tissues. The rates of strong-positive expression were 2.5\% (2/80) and 25\% (20/80), respectively. There was significantly higher expression 

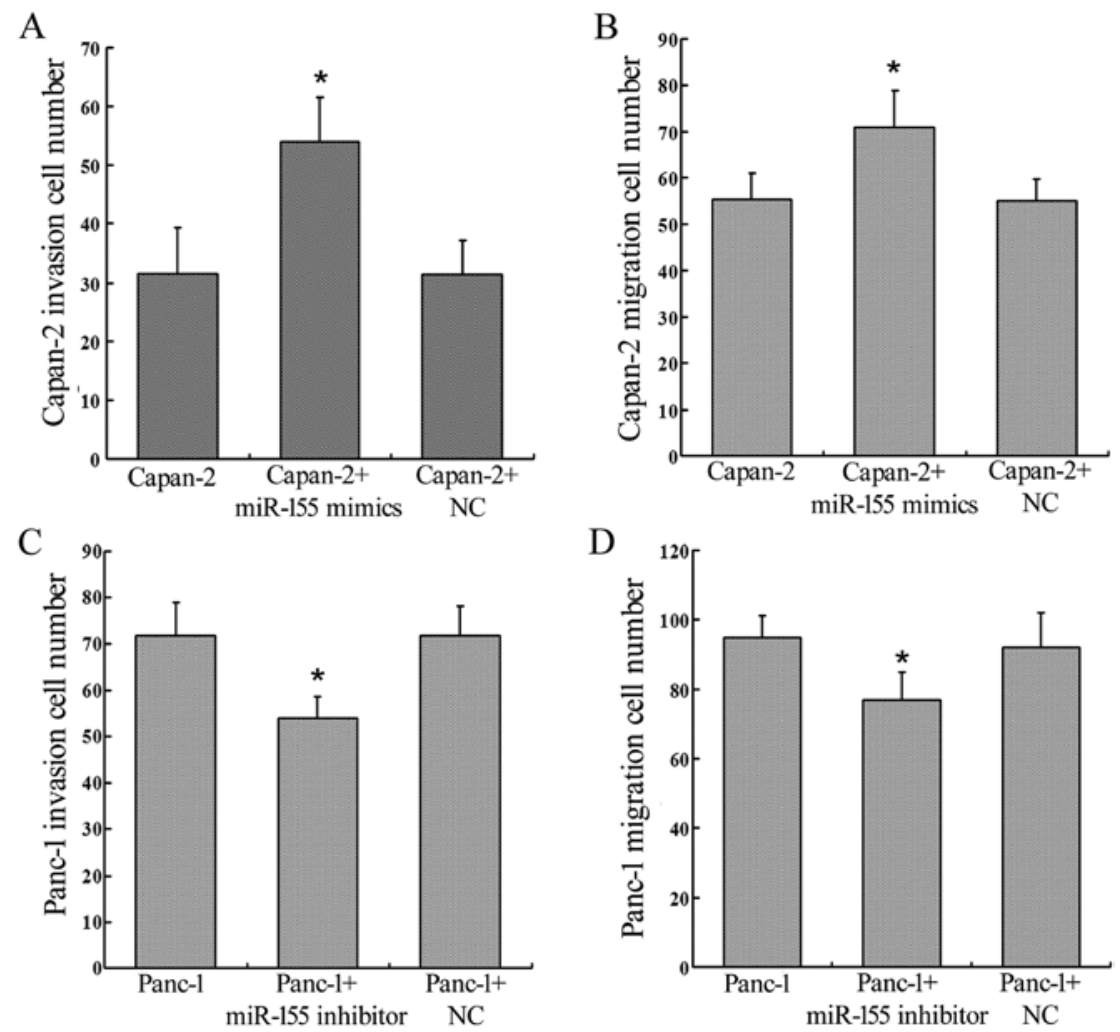

Figure 3. The number of invasive and migratory pancreatic cancer cells in vitro. (A and B) Upregulation of expression of miR-155 in Capan-2 cells enhanced the number of invasive and migratory cells in vitro ("P<0.01). (C and D) Knockdown of expression of miR-155 in Panc-1 cells decreased the number of invasive and migratory cells $\left({ }^{*} \mathrm{P}<0.01\right)$.

A
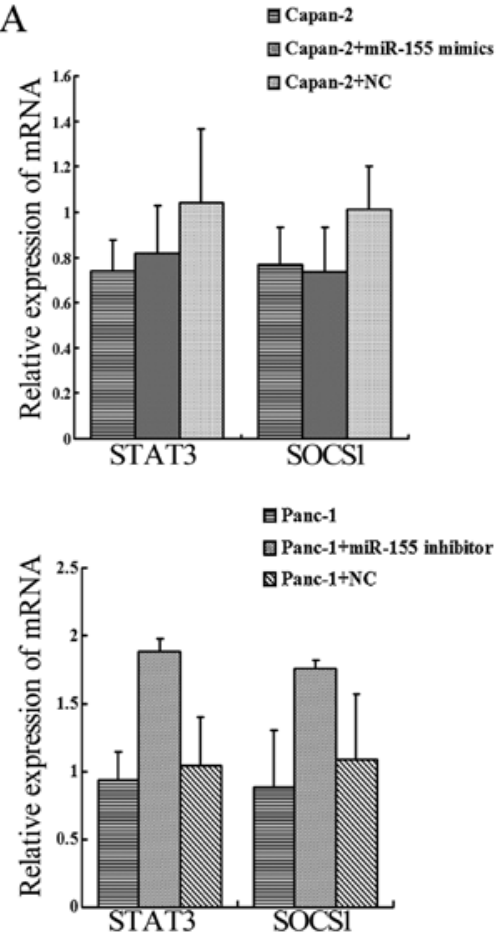

B

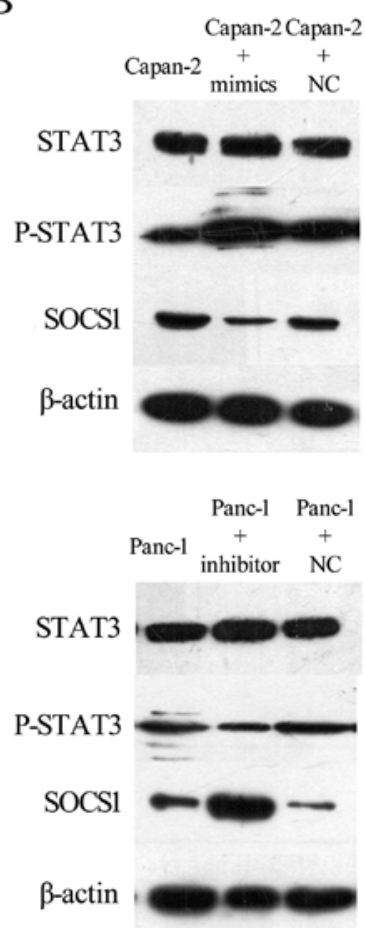

Figure 4. Expression of suppressor of cytokine signaling 1 (SOCS1) and signal transducer and activator of transcription-3 (STAT3) and activation of STAT3 following regulation of miR-155. (A) SOCS1 and STAT3 mRNA expression did not significantly differ (<2-fold or 50\%) in the transfected cells when compared to the parental and control cells. (B) SOCS1 protein expression increased when miR-155 expression was knocked down in Panc-1 cells while SOCS1 protein expression decreased following miR-155 upregulation; the opposite pattern was observed for P-STAT3 protein.

in the tumor-adjacent tissues when compared with that in the cancer tissues $(\mathrm{P}=0.0003)$ (Fig. 5B) (Table II).
Relationship between miR-155 and SOCS1 expression in pancreatic cancer and tumor-adjacent tissues. We analyzed 
Table I. Expression of miR-155 in pancreatic tumor and tumor-adjacent tissues.

\begin{tabular}{|c|c|c|c|c|c|}
\hline & \multicolumn{4}{|c|}{ miR-155 } & \multirow[b]{2}{*}{ Positive rate $(\%)$} \\
\hline & - & + & ++ & +++ & \\
\hline \multicolumn{6}{|l|}{ Tissue } \\
\hline Tumor & 15 & 27 & 30 & 8 & $81.5^{\mathrm{a}}$ \\
\hline Tumor-adjacent & 23 & 1 & 55 & 1 & 71.25 \\
\hline
\end{tabular}

${ }^{\mathrm{a}} \mathrm{P}<0.01$ vs. tumor-adjacent tissue.
Table II. Expression of SOCS1 protein in pancreatic tumor and tumor-adjacent tissues.

\begin{tabular}{lccccc}
\hline & \multicolumn{4}{c}{ SOCS1 protein } & \\
\cline { 2 - 4 } & - & + & ++ & +++ & Positive rate $(\%)$ \\
\hline Tissue & 50 & 17 & 11 & 2 & $37.5^{\mathrm{a}}$ \\
Tumor & 28 & 17 & 15 & 20 & 65 \\
Tumor-adjacent & 28 & & & & \\
\hline
\end{tabular}

${ }^{\mathrm{a}} \mathrm{P}<0.01$ vs. tumor-adjacent tissue. SOCS 1 , suppressor of cytokine signaling 1 .

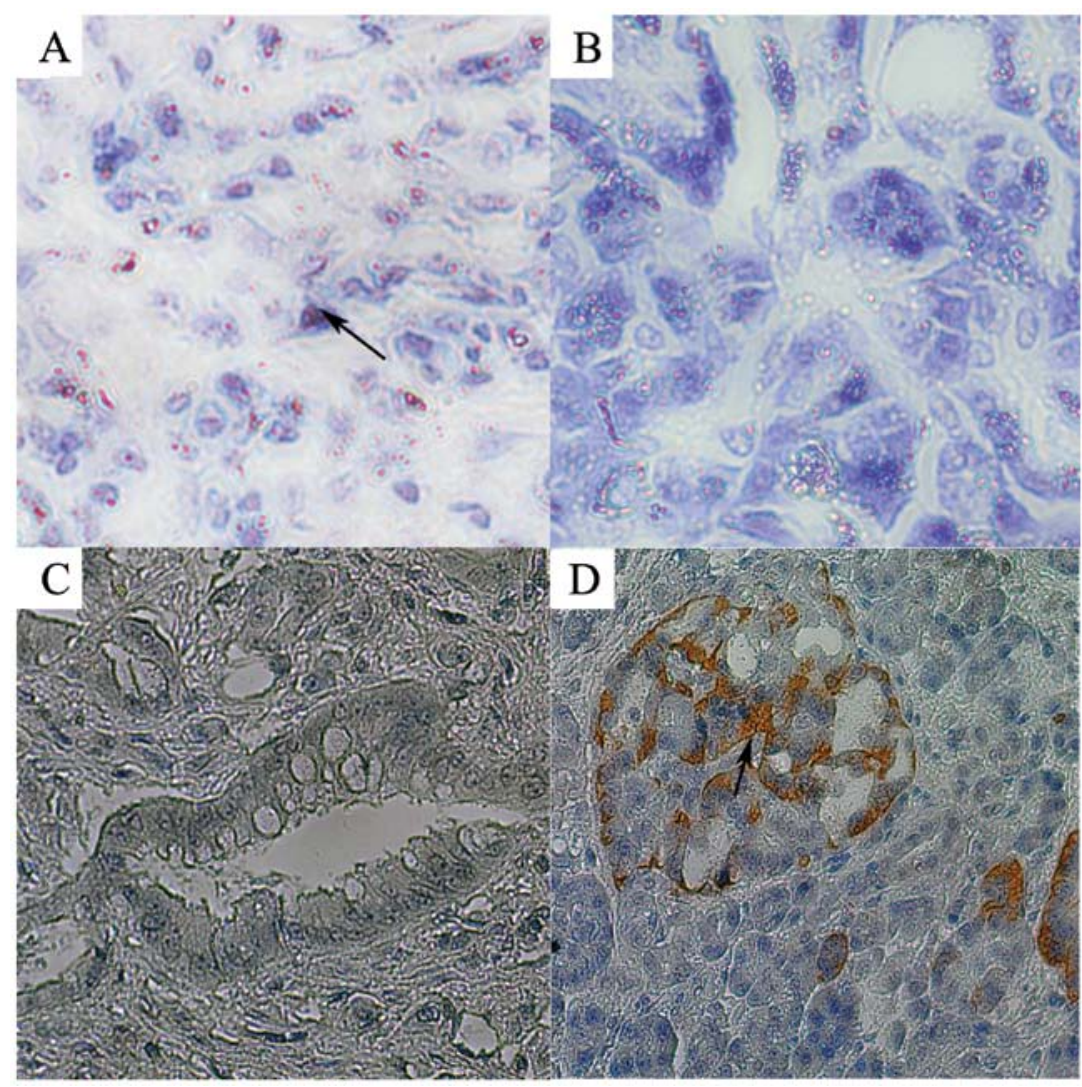

Figure 5. Expression of miR-155 and suppressor of cytokine signaling 1 (SOCS1) in pancreatic cancer and tumor-adjacent tissues. (A) miR-155 expression in pancreatic cancer tissue. miR-155 expression was noted in cancer cells (black arrow). (B) miR-155 expression in tumor-adjacent tissue. (C) SOCS1 expression in pancreatic cancer tissue. (D) SOCS1 expression in tumor-adjacent tissue. SOCS1 expression was noted in normal pancreatic ductal epithelial cells (black arrow).

the relationship between miR-155 and SOCS1 expression in pancreatic cancer and tumor-adjacent tissues. The data revealed no relationship between miR-155 and SOCS1 in cancer or tumor-adjacent tissues $\left(\mathrm{r}_{1}=-0.178, \mathrm{P}_{1}=0.115\right.$; $\left.r_{2}=-0.002, P_{2}=0.947\right)$ (Tables III and IV).

Relationship between miR-155, SOCS1 and clinical stage of pancreatic cancer. We analyzed the relationship between miR-155, SOCS1 and the clinical stage of pancreatic cancer and found SOCS1-positive expression in 33.3\% (13/39) of the non-lymph node metastatic pancreatic cancer tissues and in $48.4 \%(15 / 31)$ of the lymph node metastatic pancreatic cancer tissues. Thus, positive expression of SOCS1 was not related to lymph node metastasis $(\mathrm{P}=0.767)$. Positive expression was noted in $34.5 \%$ (10/29), 22.22\% (2/9), 34.37\% (11/32) of stage I, IIa and IIb + III + IV cases, respectively. However, there was no relationship between positivity and clinical stage $(\mathrm{P}=0.539)$ (Table V).

Positive expression of miR-155 was noted in 30.8\% (12/39) of the non-lymph node metastatic pancreatic cancer tissues and in $67.7 \%(21 / 31)$ of the lymph node metastatic pancreatic cancer tissues. Thus, miR-155 expression was related to lymph node metastasis $(\mathrm{P}=0.0001)$. Positive expression was noted in $51.7 \%(15 / 29), 55.5 \%(5 / 9)$ and $81.25 \%(26 / 32)$ of stage I, IIa and IIb + III + IV cases, respectively. Thus, miR-155 expression was related to clinical stage $(\mathrm{P}=0.011)$ (Table VI). 
Table III. miR-155 and SOCS1 expression in pancreatic cancer tumors.

\begin{tabular}{|c|c|c|c|c|c|c|}
\hline & \multicolumn{4}{|c|}{ miR-155 } & \multicolumn{2}{|c|}{$\begin{array}{c}\text { Spearman's rank } \\
\text { correlation }\end{array}$} \\
\hline & $\begin{array}{c}- \\
(n=15)\end{array}$ & $\begin{array}{c}+ \\
(n=27)\end{array}$ & $\begin{array}{c}++ \\
(\mathrm{n}=30)\end{array}$ & $\begin{array}{c}+++ \\
(n=8)\end{array}$ & $\mathrm{r}$ & $\mathrm{P}$-value \\
\hline SOCS1 & & & & & -0.178 & 0.115 \\
\hline$-\quad(n=50)$ & 8 & 14 & 23 & 5 & & \\
\hline$+\quad(n=17)$ & 4 & 7 & 4 & 2 & & \\
\hline$++\quad(n=11)$ & 2 & 5 & 3 & 1 & & \\
\hline$+++(n=2)$ & 1 & 1 & 0 & 0 & & \\
\hline
\end{tabular}

SOCS1, suppressor of cytokine signaling 1 .

Table IV. miR-155 and SOCS1 expression in tumor-adjacent tissue.

\begin{tabular}{|c|c|c|c|c|c|c|}
\hline & \multicolumn{4}{|c|}{ miR-155 } & \multicolumn{2}{|c|}{$\begin{array}{c}\text { Spearman's ran } \\
\text { correlation }\end{array}$} \\
\hline & $\begin{array}{c}- \\
(n=23)\end{array}$ & $\begin{array}{c}+ \\
(n=1)\end{array}$ & $\begin{array}{c}++ \\
(n=55)\end{array}$ & $\begin{array}{c}+++ \\
(\mathrm{n}=1)\end{array}$ & $\mathrm{r}$ & P-value \\
\hline SOCS1 & & & & & -0.002 & 0.947 \\
\hline$-\quad(n=28)$ & 6 & 0 & 22 & 0 & & \\
\hline$+\quad(n=17)$ & 8 & 0 & 8 & 1 & & \\
\hline$++\quad(n=15)$ & 4 & 0 & 11 & 0 & & \\
\hline$+++(n=20)$ & 5 & 1 & 14 & 0 & & \\
\hline
\end{tabular}

SOCS1, suppressor of cytokine signaling 1.

\section{Discussion}

miR-155 mimics and inhibitor respectively upregulated and downregulated expression of miR-155 in Panc-1 and Capan-2 cells in comparison to the parental and negative control cells. Invasion and migration ability of pancreatic cancer cells was significantly reduced in vitro when miR-155 was downregulated. The reverse was true for miR-155 upregulation. miR-155 was previously found to be highly expressed in pancreatic cancer tissues $(19,20)$ and to influence invasion and metastasis through its target genes including TP53INP1 and RhoA $(13,14)$. Our results are consistent with these previous reports. However, our study was performed in vitro; in vivo studies will follow.

miRNAs have hundreds of potential target genes. SHIP1, $\mathrm{C} / \mathrm{EBP} \beta$ and CK1 $\alpha$ are targets of miR-155 (21-23). As these genes have different functions, miR-155 plays multiple roles in cancer development. Reports indicate that SOCS1 is a target gene of miR-155 in breast cancer and plays an important role in activation of the STAT3 signaling pathway (24). In the present study, we found that expression of SOCS1 protein (not transcription) in pancreatic cancer cells was regulated by miR-155. This finding suggests that miR-155 regulated SOCS1 expression only at the subtranscription level but did not lead to its mRNA degradation. Phosphorylation of STAT3 was also affected by
Table V. Distribution of SOCS1 expression in pancreatic cancer tumors according to TNM stage and lymph node metastasis.

\begin{tabular}{lrrrr}
\hline & & \multicolumn{3}{c}{ SOCS1 } \\
\cline { 3 - 5 } & $\mathrm{n}$ & Low & High & P-value \\
\hline TNM stage & & & & 0.767 \\
I & 29 & 19 & 10 & \\
IIa & 9 & 7 & 2 & \\
IIb + III + IV & 32 & 21 & 11 & \\
Lymph nodes & & & & \\
No metastasis & 39 & 26 & 13 & \\
Metastasis & 31 & 16 & 15 & \\
\hline
\end{tabular}

SOCS1, suppressor of cytokine signaling 1 .

Table VI. Distribution of miR-155 expression in pancreatic cancer tumors according to TNM stage and lymph node metastasis.

\begin{tabular}{lrrrr}
\hline & & \multicolumn{3}{c}{ miR-155 } \\
\cline { 3 - 5 } & $\mathrm{n}$ & Low & High & P-value \\
\hline TNM stage & & & & \\
I & 29 & 14 & 15 & $0.000^{\mathrm{a}}$ \\
IIa & 9 & 4 & 5 & \\
IIb + III + IV & 32 & 6 & 26 & \\
Lymph nodes & & & & $0.011^{\mathrm{a}}$ \\
No metastasis & 39 & 27 & 12 & \\
Metastasis & 31 & 10 & 21 & \\
\hline
\end{tabular}

${ }^{\mathrm{a}} \mathrm{P}<0.01$.

miR-155, but with a reverse trend. Thus, miR-155 may influence pancreatic cancer invasion and metastasis, at least partly, by regulating SOCS1 through the STAT3 signaling pathway. Our previous study showed that overactivation of the STAT3 signaling pathway in pancreatic cancer regulated MMP-2, MMP-7 and others to mediate invasion and metastasis $(18,25)$. However, we do not know what induces the overactivation of STAT3 in pancreatic cancer. We now suggest that high miR-155 expression may be one cause of STAT3 overactivation in pancreatic cancer. Other reports have indicated that miR-155 activates the STAT3 signaling pathway in cancer cells $(26,27)$. However, these hypotheses require in vivo validation.

Reports indicate that expression of miR-155 and SOCS1 is related to clinical stage and prognosis (28-30). We found increased expression of miR-155 in pancreatic cancer tissue, but not in tumor-adjacent tissues. SOCS1 was more highly expressed in tumor-adjacent tissues than in pancreatic cancer tissues. However, there was no relationship between these phenomena. This may suggest some contradiction with our in vitro results. However, we know that expression of one type of protein may be regulated by many factors and there is a reticular structure to the regulation of protein expression. Therefore, of the many factors influencing SOCS1 expression in pancreatic cancers, $\mathrm{miR}-155$ is one. 
Our study also showed that expression of miR-155 was related to lymph node metastasis and clinical stage. A previous study indicated that miR-155 levels significantly increased in intraepithelial neoplasia grade II pancreatic ductal epithelial cells or early-stage pancreatic cancer in comparison to normal pancreatic tissues (31). This result suggests that when malignant transformation occurs in pancreatic ductal epithelial cells, miR-155 levels increase. Several studies have shown increased miR-155 expression in pancreatic cancer tissues in comparison to normal pancreatic tissues or chronic pancreatitis tissues, and they showed that miR-155 may serve as an index for diagnosis and clinical staging $(12,32,33)$. These results are consistent with our study. However, other studies have indicated that miR-155 can inhibit gastric cancer invasion and metastasis by altering expression of smad2, acting as a type of tumorsuppressor gene (34). Therefore, continued investigation of the roles of miR-155 in pancreatic cancer and its relationship with metastasis and prognosis is warranted.

Identification of the molecular mechanisms responsible for pancreatic cancer invasion and metastasis are critical to successful treatment of this disease. In the present study, we found that miR-155 can affect activation of STAT3 to mediate invasion and metastasis through SOCS1. These findings may be helpful to find suitable targets for microRNA-based gene therapy and for novel approaches for the early diagnosis of pancreatic cancer.

\section{Acknowledgements}

The present study was supported by grants 81101844 and 81210108027 (to C.H.) from the National Natural Science Foundation of China and grants 2012040 and 13PJD024 (to C.H.) from Shanghai Municipal Human Resources and Social Security Bureau.

\section{References}

1. Stefani G and Slack FJ: Small non-coding RNAs in animal development. Nat Rev Mol Cell Biol 9: 219-230, 2008.

2. Jemal A, Bray F, Center MM, et al: Global cancer statistics. CA Cancer J Clin 61: 69-90, 2011.

3. Yu J, Li A, Hong SM, et al: MicroRNA alterations of pancreatic intraepithelial neoplasias. Clin Cancer Res 18: 981-992, 2012.

4. Chen Z, Chen LY, Dai HY, et al: miR-301a promotes pancreatic cancer cell proliferation by directly inhibiting Bim expression. J Cell Biochem 113: 3229-3235, 2012.

5. Tam W, Ben-Yehuda D and Hayward WS: bic, a novel gene activated by proviral insertions in avian leukosis virus-induced lymphomas, is likely to function through its noncoding RNA. Mol Cell Biol 17: 1490-1502, 1997.

6. Kong W, He L, Coppola M, et al: MicroRNA-155 regulates cell survival, growth, and chemosensitivity by targeting FOXO3a in breast cancer. J Biol Chem 285: 17869-17879, 2010.

7. Babar IA, Czochor J, Steinmetz A, Weidhaas JB, et al: Inhibition of hypoxia-induced miR-155 radiosensitizes hypoxic lung cancer cells. Cancer Biol Ther 12: 908-914, 2011.

8. Bakirtzi K, Hatziapostolou M, Karagiannides I, et al: Neurotensin signaling activates microRNAs-21 and -155 and Akt, promotes tumor growth in mice, and is increased in human colon tumors. Gastroenterology 141: 1749-1761, 2011.

9. Nikiforova MN, Tseng GC, Steward D, et al: MicroRNA expression profiling of thyroid tumors: biological significance and diagnostic utility. J Clin Endocrinol Metab 93: 1600-1608, 2008.

10. Szafranska AE, Davison TS, John J, et al: MicroRNA expression alterations are linked to tumorigenesis and non-neoplastic processes in pancreatic ductal adenocarcinoma. Oncogene 26: 4442-4452, 2007.
11. Bloomston M, Frankel WL, Petrocca F, et al: MicroRNA expression patterns to differentiate pancreatic adenocarcinoma from normal pancreas and chronic pancreatitis. JAMA 297: 1901-1908, 2007.

12. Greither T, Grochola LF, Udelnow A, et al: Elevated expression of microRNAs 155, 203, 210 and 222 in pancreatic tumors is associated with poorer survival. Int J Cancer 126: 73-80, 2010.

13. Gironella M, Seux M, Xie MJ, et al: Tumor protein 53-induced nuclear protein 1 expression is repressed by miR-155, and its restoration inhibits pancreatic tumor development. Proc Natl Acad Sci USA 104: 16170-16175, 2007.

14. Kong W, Yang H, He L, et al: MicroRNA-155 is regulated by the transforming growth factor beta/Smad pathway and contributes to epithelial cell plasticity by targeting RhoA. Mol Cell Biol 28: 6773-6784, 2008.

15. Davey GM, Heath WR and Starr R: SOCS1: a potent and multifaceted regulator of cytokines and cell-mediated inflammation. Tissue Antigens 67: 1-9, 2006.

16. Zhang J, Zhao H, Chen J, et al: Interferon- $\beta$-induced miR-155 inhibits osteoclast differentiation by targeting SOCS1 and MITF. FEBS Lett 586: 3255-3262, 2012.

17. Huang C, Yang G, Jiang T, et al: The effects and mechanisms of blockage of STAT3 signaling pathway on IL-6 inducing EMT in human pancreatic cancer cells in vitro. Neoplasma 58: 396-405, 2011.

18. Li HD, Huang C, Huang KJ, et al: STAT3 knockdown reduces pancreatic cancer cell invasiveness and matrix metalloproteinase-7 expression in nude mice. PLoS One 6: e25941, 2011.

19. Lee EJ, Gusev Y, Jiang J, et al: Expression profiling identifies microRNA signature in pancreatic cancer. Int J Cancer 120: 1046-1054, 2007.

20. Habbe N, Koorstra JB, Mendell JT, et al: MicroRNA miR-155 is a biomarker of early pancreatic neoplasia. Cancer Biol Ther 8: 340-346, 2009.

21. Pedersen IM, Otero D, Kao E, et al: Onco-miR-155 targets SHIP1 to promote TNFalpha-dependent growth of B cell lymphomas. EMBO Mol Med 1: 288-295, 2009.

22. Costinean S, Sandhu SK, Pedersen IM, et al: Src homology 2 domain-containing inositol-5-phosphatase and CCAAT enhancer-binding protein $\beta$ are targeted by miR-155 in B cells of E $\mu$-MiR-155 transgenic mice. Blood 114: 1374-1382, 2009.

23. Zhang P, Bill K, Liu J, Young E, et al: MiR-155 is a liposarcoma oncogene that targets casein kinase- $1 \alpha$ and enhances $\beta$-catenin signaling. Cancer Res 72: 1751-1762, 2012.

24. Jiang S, Zhang HW, Lu MH, et al: MicroRNA-155 functions as an OncomiR in breast cancer by targeting the suppressor of cytokine signaling 1 gene. Cancer Res 70: 3119-3127, 2010.

25. Huang C, Cao J, Huang KJ, et al: Inhibition of STAT3 activity with AG490 decreases the invasion of human pancreatic cancer cell in vitro. Cancer Sci 97: 1417-1423, 2006.

26. Jiang S, Zhang LF, Zhang HW, et al: A novel miR-155/miR-143 cascade controls glycolysis by regulating hexokinase 2 in breast cancer cells. EMBO J 31: 1985-1998, 2012.

27. Su C, Hou Z,Zhang C, et al: Ectopic expression of microRNA-155 enhances innate antiviral immunity against HBV infection in human hepatoma cells. Virol J 8: 354, 2011.

28. Han ZB, Chen HY, Fan JW, et al: Up-regulation of microRNA-155 promotes cancer cell invasion and predicts poor survival of hepatocellular carcinoma following liver transplantation. J Cancer Res Clin Oncol 138: 153-161, 2012.

29. Sasi W, Jiang WG, Sharma A and Mokbel K: Higher expression levels of SOCS 1,3,4,7 are associated with earlier tumour stage and better clinical outcome in human breast cancer. BMC Cancer 10: 178,2010

30. Zhang J, Li H, Yu JP, et al: Role of SOCS1 in tumor progression and therapeutic application. Int J Cancer 130: 1971-1980, 2012.

31. Ryu JK, Hong SM, Karikari CA, et al: Aberrant microRNA-155 expression is an early event in the multistep progression of pancreatic adenocarcinoma. Pancreatology 10: 66-73, 2010.

32. Panarelli NC, Chen YT, Zhou XK, et al: MicroRNA expression aids the preoperative diagnosis of pancreatic ductal adenocarcinoma. Pancreas 41: 685-690, 2012.

33. Wang J, Chen J, Chang P, et al: microRNAs in plasma of pancreatic ductal adenocarcinoma patients as novel blood-based biomarkers of disease. Cancer Prev Res 2: 807-813, 2009.

34. Li CL, Nie H, Wang M, et al: microRNA-155 is downregulated in gastric cancer cells and involved in cell metastasis. Oncol Rep 27: 1960-1966, 2012. 